\title{
An immediate and permanent cure for Puberphonia
}

\section{Short communication}

Persistence of child voice (feminine) in male adult is called Puberphonia. The voice is characterized by a weak quality and small dynamic range.

Men's voices may be said to an average in range from bass (E 80 d.v. to E 320 d.v.) to tenor (C 128 d.v. to B 480 d.v.) Women voice an average from contralto (E 160 d.v. to E 640 d.v.) to soprano (C 256 d.v. to C 1024 d.v.).

In the male the increase in size of the larynx at puberty deepens the pitch of the voice and, because of the relative suddenness of the change; control for a time is imperfect. The larger larynx includes longer cords and deeper pitch. The voice may break or it may alternate between a higher and lower pitch. In girls the growth of the larynx is more gradual and there is much less difference in pitch between the voice of girlhood and womanhood.

Similarly voice of the aged human being is different; the shrill and tremulous voice of many persons in the eighth decade is due to structural changes in the cords and feebleness of nervous and muscular energy. The senile changes in the cords are fibrosis and hyalinization resulting in a coarser, denser connective tissue with an increase in the squamous epithelial covering. Though these changes are more or less normal for age, repeated inflammatory attacks through the years are probably contributory factors. Senile changes in epithelium probably supply a favorable soil for a growth of cancer of the larynx.

\section{New technique in management}

The new technique in management: As soon as the patient is received a complete examination is done to exclude local pathology. None of the cases have local lesion. Complete ENT examination is normal. The structure and function of both vocal cords are normal. The procedure is done as an outpatient procedure and he is taken to the outpatient theater. Under local anaesthesia (mouth and throat spray with $4 \%$ xylocane) tongue depressor is used to see the uvula clearly with a curved long artery forceps uvula is cached and ask him to say few words with open mouth. Slowly he will get the masculine voice. It is continued with repetition. Along the artery forceps a tie is placed with silk thread and the artery forceps is removed.

He is requested to shout with open mouth. The voice should come from soft palate, not from the larynx. He can continue to practice with the thread inside the mouth. Regular voice therapy makes a permanent male voice. The important of voice therapy: The voice should come from the tooth, lip, tongue, nose and palate. Not from the larynx.

\section{Concept of this therapy}

500 years back Tholkappiyar clearly told about the origin of voice and production voice and how to strengthen the voice. Voice should come from lower abdomen the sound should vibrate and resonate in the head, neck and chest. The voice should be delivered out only by tooth, lip, tongue, nose and palate. So palatal voice should be practiced before this surgical procedure. Patient is encouraged to stand in front of the mirror and practice to make loud noice with open mouth with the movement of the soft palate.

\author{
Volume 10 Issue 4 - 2018 \\ Kumaresan Muthiah \\ ENT Surgeon, Siva ENT Head and Neck Hospital, India
}

Correspondence: Kumaresan Muthiah, ENT Surgeon, Siva ENT Head And Neck Hospital, 94(New No: I59), Avvai Shanmugam Salai (Lloyds' Road), Royapettah, Chennai- 600014 , India, Tel (044) 28I I6807, +9I 9841055774,

Email kumaresan@doctor.com

Received: April 23, 2017| Published: July 31, 2018

For this three types of forcible expiration is done with loud noice.1, two arms should be stretched forward and with inspiration, hand is flexed to the chest and with open mouth. Then make loud voice with palate along with forceful streching out of arms: 2. Move the hand downward, with inspiration flex the hand to the chest and make loud noise while expiration with open mouth and movement of the soft palate, stretch out the arms forcefully downwards. 3. Similarly the hand should be raised above the head and then bring hand to the chest with inspiration. Forcefully stretch upward the hand with open mouth shouting done with palate movement.

The same things should be repeated several times. For 1 to 2 hours patient should shout with his high voice. It will become a permanent adult male voice. Out of eleven cases six cases had alternating high voice and puberphonia. With good encouragement next day itself these cases even picked up adult male voice and later they had been habituated into normal adult voice. Upto 5 years we have followed few cases, no recurrence of puberphonia.

\section{Conclusion}

A small technique of treatment for puberphonia is illustrated. The treatment is permanent and immediate. This treatment also completely eliminates the old theories of endocrinal imbalance, failure of development of secondary sexual characteristics, maturation disturbances, abnormal functional adaptation, deep-seated psychological problems, immature voice, chest voice, breaking voice, immaturity, fear of adult responsibility, losing maternal protection, hero worship of an older boy, feminine tendencies, rejection of masculine voice, small larynx, short vocal folds and natural tenor voice. (The typical tenor voice lies between $\mathrm{C} 3$ (one octave below middle C) to C5 (one octave above middle C). The low extreme for tenors. Singing outside the natural vocal range imposes a serious strain upon the voice.)

\section{Acknowledgments}

None.

\section{Conflict of interest}

The author declares there is no conflict of interest. 